\title{
MODELOS PARA O ENSINO DO EXAME DA CAVIDADE ORAL EM ROEDORES E COELHOS
}

\author{
(Models for teaching oral examination in rodents and rabbits)
}

\begin{abstract}
Helena Baggio Soares ${ }^{1 *}$; Rogério Ribas Lange ${ }^{1}$; Gabriela Lepasky da Cruz ${ }^{2}$; Bruna Natali da Costa ${ }^{1}$; Fernanda Naomi Tanji $;$ Simone Tostes de Oliveira Stedile ${ }^{1}$

1Programa de Pós-graduação em Ciências Veterinárias, Universidade Federal do Paraná, Curitiba, PR; ${ }^{2}$ Curso de Medicina Veterinária, Universidade Federal do Paraná, Curitiba, PR.
\end{abstract}

*Autor para correspondência: mvhelenabaggio@gmail.com

RESUMO - O exame da cavidade oral de roedores e coelhos domésticos é muito importante para o diagnóstico da síndrome do desgaste dentário inadequado, que leva ao hipercrescimento dentário e consequentemente a problemas sistêmicos. A anatomia da cavidade oral não favorece o exame, sendo indicado o uso de otoscópio para a observação dos dentes molares. O objetivo deste estudo foi criar modelos para o ensino da avaliação da cavidade oral de roedores e lagomorfos. Os modelos para avaliação com otoscópio foram confeccionados a partir de crânios de cadáveres éticos, silicone e tecido de pelúcia. Como complemento, foram confeccionados modelos de resina a partir de moldes obtidos de cadáveres e também disponibilizados sincrânios normais e alterados. Foi feita a validação dos modelos com a participação de médicos veterinários que trabalham com o otoscópio como ferramenta para avaliação da cavidade oral. Após a validação de conteúdo pelos experts, o modelo foi apresentado aos estudantes para validação de construto. Participaram do estudo 30 estudantes de medicina veterinária, que após aula teórica sobre odontologia de roedores e lagomorfos e aula prática com os modelos, responderam a um questionário. A maioria dos estudantes $(98 \%)$ considerou os modelos realistas e a prática didática e citou que os modelos auxiliaram na melhor compreensão da avaliação dentária e no melhor entendimento anatômico dos dentes de roedores e lagomorfos. Com isso, pôde-se perceber que esse tipo de modelo para estudo e treinamento da odontologia de roedores e lagomorfos foi bem aceito pelos estudantes. Pela importância do estudo da odontologia veterinária como especialidade nesse grupo específico de animais, recomendamos que os modelos para exame da cavidade oral sejam introduzidos na rotina das universidades.

Palavras-chave: modelos; odontologia; otoscópio; simulador.

ABSTRACT - The inspection of the oral cavity on domestic rodents and rabbits is extremely important for inadequate dental trimming syndrome diagnosis, that results in dental overgrowth and, consequently, systemic problems. The oral cavity anatomy interferes in the exam, therefore, using an otoscope is necessary to evaluate the cheek teeth. The aim of this project was to create a model for teaching the otoscope technique for oral cavity examination in rodents and lagomorphs. The models were designed from ethically sourced animal cadavers skulls, silicons and fake fur fabric. As a complement, resin models were made from the skulls which had oral cavity abnormalities. In addition to that, normal and abnormal syncranium were showed. For the model validation, six veterinarians that have experience with the otoscope technique were asked to evaluate the models. After the professional evaluation, the 
models were presented to students for construct validation. In this process, 30 students from the Veterinary Medicine course attended to a theoretical class about rodents and rabbits odontology. After that, all 30 students practiced on the models and completed a survey. The majority of the students $(98 \%)$ considered the models realistic and helpful to understand the technique. Also, it was pointed as important for rodents and rabbits anatomy comprehension. The models positively impacted students learning process and leading to a positive feedback. Therefore, these types of models can be an important tool for teaching and practicing rodents and lagomorphs odontology. We highly recommend the models of oral cavity exam for classes in universities, due to the importance of the odontology in this group of animals.

Palavras-chave - models; odontology; otoscope; simulator. 


\section{INTRODUÇÃO}

As Ordens Rodentia e Lagomorpha abrangem centenas de espécies (Presgrave, 2002), muitas delas domesticadas e que estão inseridas na rotina do clínico de animais selvagens, como o coelho (Oryctolagus cuniculus), o porquinho-da-índia (Cavia porcellus) e a chinchila (Chinchila lanigera). Uma das características dentárias mais importantes dessas espécies é 0 crescimento dentário contínuo, tanto de dentes incisivos quanto de dentes molariformes (Wiggs e Lobprise, 1997; Presgrave, 2002; Legendre, 2003; Lange e Schmidt, 2014). Para que haja um equilíbrio da saúde oral desses animais, a taxa de crescimento dentário deve ser mais ou menos igual à taxa de desgaste dentário; assim, os dentes se mantêm sempre do mesmo tamanho e o animal preserva a saúde oral (Legendre, 2003).

A taxa de desgaste dentário está intimamente ligada à dieta adequada dos animais, que deve promover abrasão (Klaphake, 2006; Muller et al., 2015). Também pode estar ligada a problemas como a má-oclusão dentária, que causará diminuição ou anulará o atrito entre os dentes. A má-oclusão dos dentes incisivos ou prognatismo mandibular (Jenkins, 2010) é mais comum em coelhos e normalmente está ligada à seleção artificial, em que são priorizados animais pequenos. $\mathrm{O}$ hipercrescimento dentário é parte da síndrome do desgaste inadequado (Diniz et al., 2006; Lennox, 2008; Bohmer e Crossley, 2011). É uma doença muito prevalente em roedores e lagomorfos domésticos, podendo acometer até cerca de $60 \%$ desses animais (Mueller et al., 2014). O crescimento exacerbado dos dentes tende a causar inicialmente problemas oftálmicos (Wiggs e Lobprise, 1997; Diniz et al, 2006; Lennox, 2008; Jenkins, 2010; Bohmer e Crosley, 2011; Lange e Schmidt, 2014), pois a coroa de reserva faz crescimento retrógrado, ou seja, na direção da órbita. Além disso, por conta da dificuldade do animal se alimentar, o hipercrescimento dentário causa também problemas em trato digestório (Wiggs e Lobprise, 1997; Diniz et al, 2006; Capello, 2008; Lennox, 2008; Jenkins, 2010; Bohmer e Crossley, 2011; Lange e Schmidt, 2014) e atonia (Diniz et al, 2006). Todos esses problemas podem agravar a saúde geral do animal. Uma boa avaliação dentária em toda visita desses animais à clínica veterinária pode potencialmente evitar ou reduzir o aparecimento da síndrome do desgaste inadequado.

A anatomia oral dos roedores e lagomorfos não favorece a avaliação dos dentes molariformes (Wiggs e Lobprise, 1997; Bohmer e Crossley, 2011; Lange e Schmidt, 2014). Eles possuem uma prega de mucosa localizada logo atrás dos incisivos que divide a cavidade oral em duas câmaras: anterior e posterior (Lange e Schmidt, 2014). Na câmara anterior ficam os dentes incisivos e na câmara posterior, os dentes molariformes. Portanto, a observação dos dentes molariformes deve ser feita com ajuda de endoscópio ou de otoscópio (Wiggs e Lobprise, 1997; Diniz et al, 2006; Lange e Schmidt, 2014), que possuem uma fonte de luz e magnificam as imagens observadas, facilitando a avaliação e o diagnóstico de crescimento dentário e de lesões em mucosas orais. A avaliação com otoscópio não é simples e requer habilidades e treinamento do médico veterinário, tanto para manusear o instrumento como para avaliar a cavidade oral de forma correta. O objetivo deste estudo foi criar modelos para treinamento da avaliação da cavidade oral em roedores e lagomorfos, usando o otoscópio como ferramenta, além de modelos de resina para observação da anatomia oral. 


\section{MATERIAL E MÉTODOS}

Foram confeccionados três modelos que simulassem uma situação de avaliação da cavidade oral de coelho, chinchila e porquinho-da-índia, com otoscópio, respeitando a consistência das estruturas como língua, gengiva e dentes. Foram confeccionados também moldes de resina como cópia fiel à cavidade oral do animal e também utilizados crânios reais de cadáveres éticos, com e sem alterações. Os modelos foram utilizados durante uma semana acadêmica do curso de medicina veterinária da Universidade Federal do Paraná. Foi ministrada uma aula teórica com o tema "Odontologia de Roedores e Lagomorfos" e, em seguida, os modelos puderam ser manipulados pelos alunos durante a aula prática.

Preparo dos crânios (para confecção dos modelos para uso do otoscópio e para visualização direta na prática complementar)

Foram preparados cinco crânios a partir de cadáveres éticos, ou seja, animais que foram submetidos à eutanásia no Hospital Veterinário, por doença terminal ou que tiveram morte natural. Eram os seguintes animais: uma cutia, duas chinchilas, um coelho e um porquinho-da-índia. Esses cadáveres estavam para descarte e os crânios foram então aproveitados para este estudo. A cavidade oral foi fotografada e em seguida foram feitos dois moldes negativos dos dentes e tecidos moles com silicone, um incluindo a parte superior e outro a parte inferior para posterior confecção do molde em resina. $\mathrm{Na}$ sequência, todo o tecido mole foi retirado das cabeças e as peças anatômicas foram preparadas por meio de fervura. Durante esse processo, cada crânio foi embalado individualmente para que os dentes não se perdessem e/ou se misturassem. Haviam dois crânios com alterações como hipercrescimento dentário, crescimento retrógrado de coroa de reserva e alteração óssea. Os crânios com alterações foram escolhidos para a observação e mantidos em sua forma original, após a retirada do tecido mole (Figura 1). Os crânios sem alterações foram utilizados para a confecção dos modelos para otoscopia.

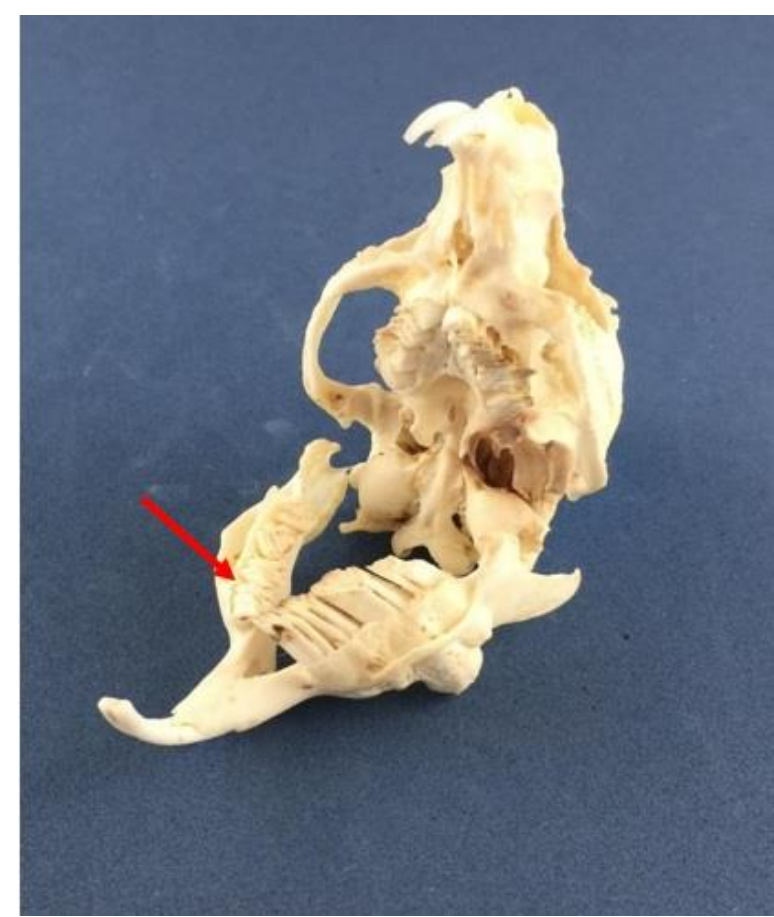

Figura 1- Crânio de porquinho-da-índia com alteração dentária (ponte dentária) (seta).

\section{Modelos para otoscopia}

Um crânio de chinchila, um de porquinho-da-índia e um de coelho sem alterações dentárias foram preparados conforme descrito anteriormente. Os crânios preparados foram a base para o modelo de otoscopia. Para confecção de língua e palato, foi esculpido molde de argila à base de óleo; estes foram copiados com silicone de média flexibilidade e novamente feito molde positivo com silicone, de densidade 0 30, adicionado de tinta até que a coloração se assemelhasse à da mucosa oral. A gengiva foi confeccionada com silicone 0-30 diretamente sobre o crânio, e as bochechas foram confeccionadas 
separadamente, com este mesmo silicone. Para recobrir todo o crânio, foram usados retalhos de tecidos de malha, simulando a pele e os pelos de cada animal (Figura 2). A abertura da boca do animal e limitação da movimentação da arcada dentária foram respeitadas, para que o uso do otoscópio no modelo tivesse as mesmas características e limitações encontradas no animal vivo (Figura 3).

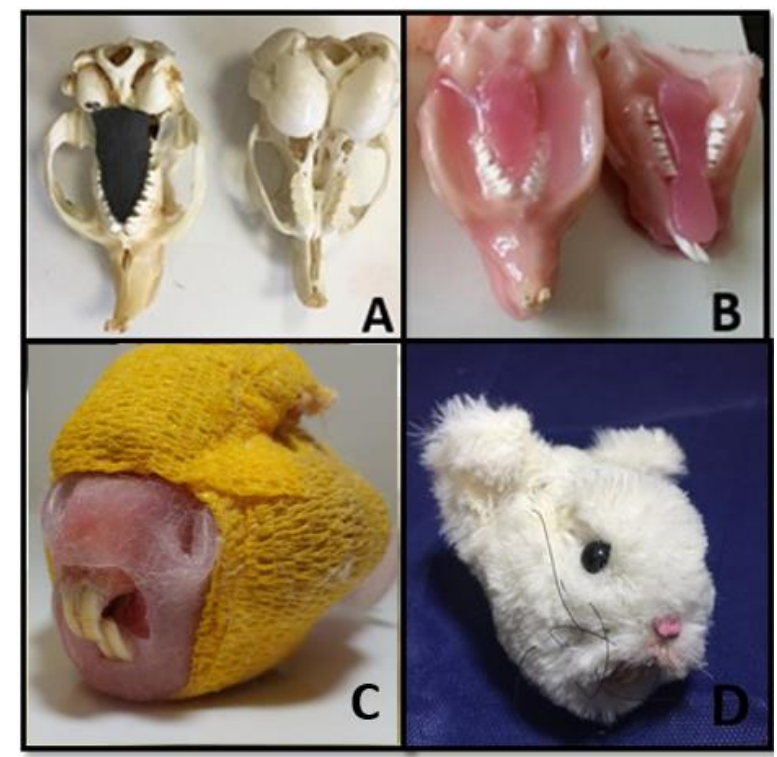

Figura 2 - Construção do modelo para visualização da cavidade oral com otoscópio. A: crânios (chinchila) e confecção do palato, esculpido com argila. B: crânios já recobertos com silicone, palato e língua já prontos. C: crânio recoberto por silicone, simulando a mucosa da bochecha. D: modelo recoberto com malha, finalizado.

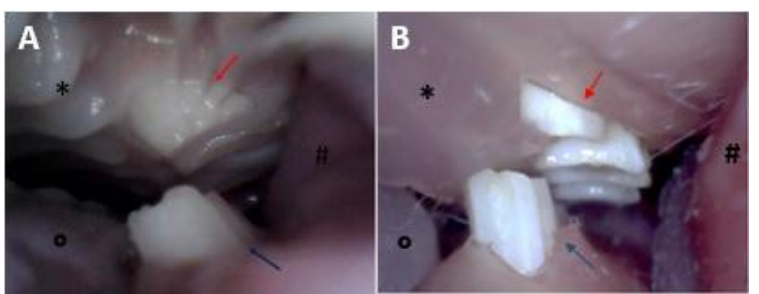

Figura 3 - Comparação da cavidade oral de um animal vivo e do modelo sintético. A: imagem de otoscópio obtida de um coelho vivo. B: imagem de otoscópio obtida do modelo de chinchila (Chinchila lanígera) usado na aula prática. As principais estruturas a se observar são: palato mole $\left(^{*}\right)$, língua $\left({ }^{\circ}\right)$, mucosa jugal $(\#)$, dente molariforme superior (flecha vermelha), dente molariforme inferior (flecha azul).

\section{Modelos de resina}

A partir do contramolde de silicone utilizado na cavidade oral do cadáver, foram feitas cópias de resina de cavidades orais normais e com alterações. Os moldes de resina foram pintados com tinta acrílica, baseando-se nas fotos da cavidade oral do cadáver (Figura 4).

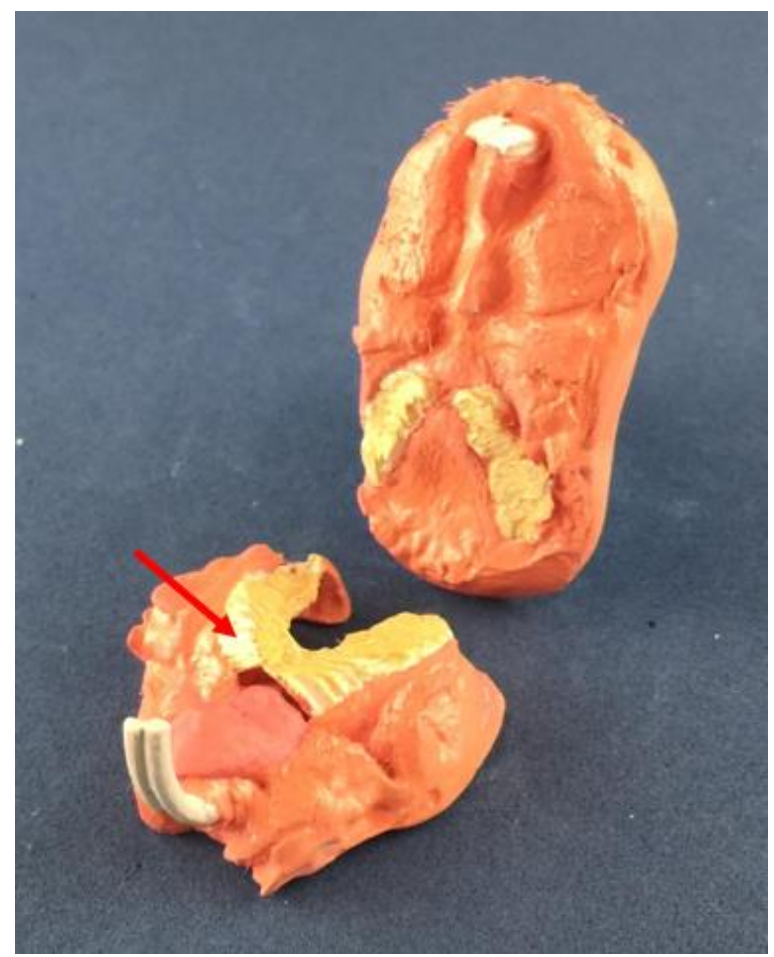

Figura 4 - Modelo de resina de cavidade oral de um porquinho-da-índia (Cavia porcellus) com hipercrescimento dentário e ponte dentária (seta) aprisionando a língua. Esse molde foi feito no animal ao qual pertence o crânio mostrado na figura 1.

\section{Validação dos modelos por profissionais da área}

Os modelos de cavidade oral para treinamento com otoscópio foram testados por seis médicos veterinários odontólogos ou de animais selvagens, com experiência na área, cuja rotina incluísse a realização do exame com otoscópio da cavidade oral de roedores e lagomorfos. Os médicos veterinários consideraram que os modelos eram adequados à prática e treinamento do exame da cavidade oral. Passou-se, então, para a etapa seguinte, para 
verificar a utilidade dos modelos em aula.

\section{Avaliação dos modelos pelos estudantes}

Foi ministrada uma palestra sobre odontologia de roedores e lagomorfos por uma médica veterinária especialista em odontologia veterinária (H.B.S.), com duração de 50 minutos, para 30 estudantes do curso de Medicina Veterinária. $\mathrm{Na}$ sequência, os estudantes tiveram aula prática com os modelos. $\mathrm{Na}$ aula prática, todos puderam observar os crânios, os moldes em resina e também praticar o exame da cavidade oral com otoscópio nos modelos de coelho, chinchila e porquinho-da-índia. Após a aula prática, foi solicitado aos estudantes que respondessem a um questionário anônimo sobre a opinião deles em relação à utilidade dos modelos no aprendizado. O questionário continha 10 perguntas sobre os modelos utilizados (modelo para uso do otoscópio, modelos em resina e crânios). Em seis dessas questões foi utilizada a escala Likert de cinco pontos (discordo fortemente, discordo, neutro, concordo, concordo fortemente) para se avaliar a percepção dos estudantes. Três questões eram abertas, em que os estudantes poderiam escrever pontos fracos e fortes de cada modelo e uma das questões era de múltipla escolha. A questão de múltipla escolha possibilitava ao estudante marcar o tipo de técnica que ele considerasse melhor ao seu aprendizado: animal vivo, modelos, cadáver e suas associações.

\section{RESULTADOS}

Fizeram parte do estudo 30 estudantes de Medicina Veterinária. Após a participação dos estudantes em uma aula teórica e uma aula prática (Figura 5) de odontologia de roedores e lagomorfos, estes responderam ao questionário sobre $o$ uso do modelo para treinamento de exame oral com otoscópio (Tabela 1), dos modelos de resina e os dos crânios. Todos os estudantes concordaram que o modelo para avaliação com o uso do otoscópio era realista e que o método de ensino com o modelo foi didático. Todos os estudantes concordaram também que o modelo permitiu compreensão tanto da avaliação da cavidade oral quanto da anatomia dentária.

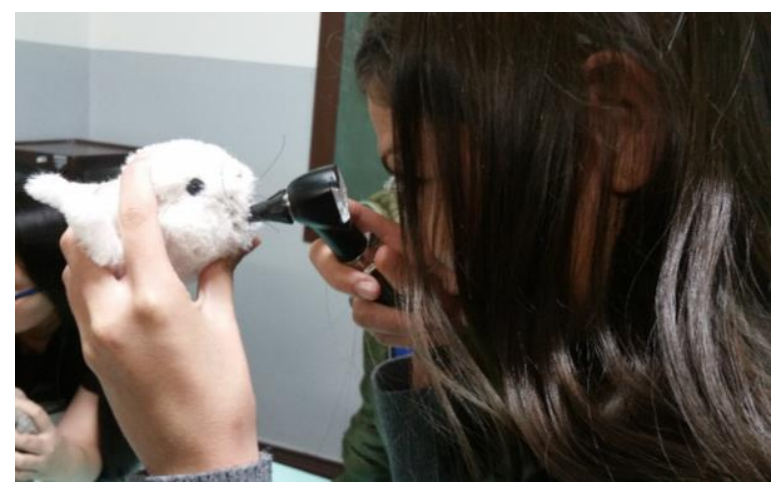

Figura 5 - Estudante avaliando a cavidade oral no modelo de chinchila, com o auxílio de otoscópio, durante a aula prática.

Tabela 1 - Respostas quantitativas dos estudantes sobre a aula prática de avaliação da cavidade oral de roedores e lagomorfos com o auxílio do otoscópio.

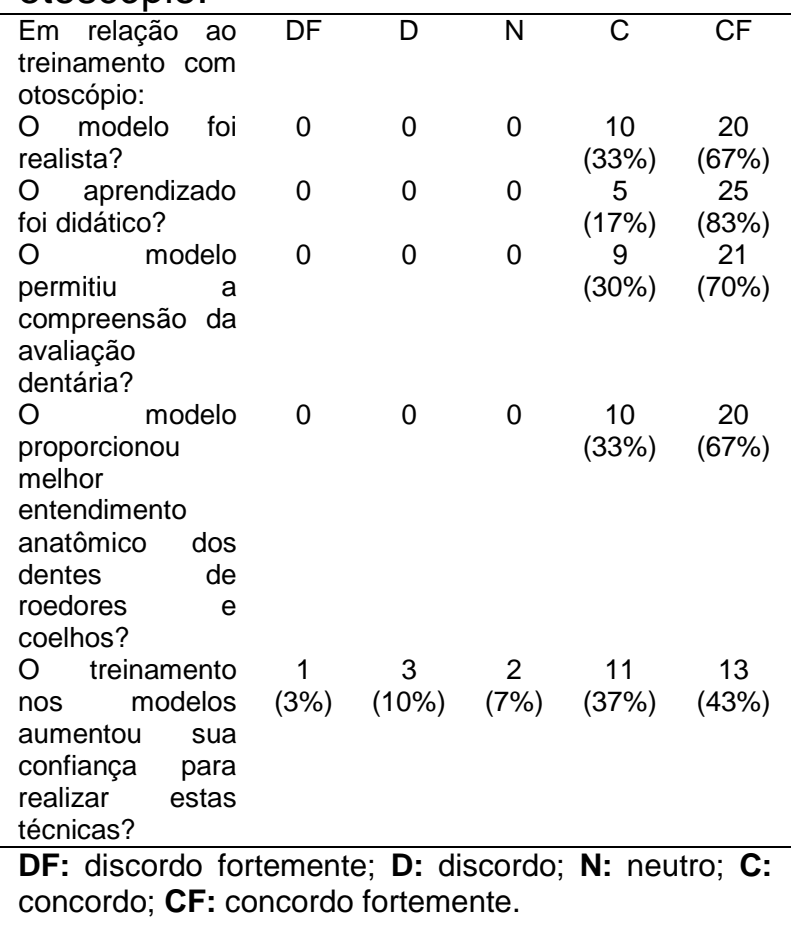


Quanto aos modelos de resina, 28 estudantes (93\%) concordaram ou concordaram fortemente com o fato de os modelos serem realistas. Vinte e sete $(90 \%)$ concordaram ou concordaram fortemente que esses modelos foram considerados um método didático para o aprendizado. A maioria (93\%) dos estudantes respondeu concordar ou concordar fortemente que os modelos de resina tenham permitido compreensão tanto da avaliação quanto da anatomia dentária.

Para os crânios houve uma aceitação de 97\%. Apenas um estudante $(3 \%)$ respondeu discordar da eficácia dos crânios no aprendizado. Vinte e nove (97\%) estudantes responderam positivamente em relação à didática dos crânios como modelo. Vinte e nove estudantes (97\%) concordaram ou concordaram fortemente que 0 modelo tenha permitido uma compreensão da avaliação dentária. Quanto ao entendimento anatômico dos dentes de roedores e lagomorfos, $100 \%$ dos estudantes respondeu concordar ou concordar fortemente.

Quando perguntados se 0 treinamento nos modelos (modelos com otoscópio, modelos em resina e crânios) aumenta a confiança para posterior realização da técnica em animais vivos, a maioria dos estudantes concordou com a afirmação. No entanto, quatro estudantes (13\%) discordaram ou discordaram fortemente.

Quando perguntados sobre as técnicas que poderiam contribuir para seu aprendizado, 28 estudantes (93\%) apontaram como sendo melhor a associação de técnicas: uso de modelos, de cadáveres e dos animais vivos. Um estudante (3\%) respondeu achar melhor 0 uso apenas de cadáveres e modelos e um estudante (3\%) respondeu achar ideal apenas o uso do modelo.
Quanto às questões abertas, os estudantes puderam dar sua opinião sobre o que perceberam como pontos fracos e fortes de cada tipo de modelo utilizado (Tabela 2). A maioria dos estudantes $(90 \%)$ comentou pontos positivos a respeito dos modelos usados em aula prática.

Tabela 2 - Comentários dos estudantes a respeito dos modelos usados em aula prática.

1. Pontos positivos

2. - Modelo próximo do real. (43,3\%)

3. - Com o modelo, podemos ter uma melhor noção e compreensão das estruturas e anormalidades. (13,3\%)

4. - Nos permite manusear e treinar bastante e nos dá mais segurança para a prática em animal vivo. (10\%)

5. - Ajudou no aprimoramento do aprendizado para somente depois utilizar a técnica em animal vivo. $(3,3 \%)$

6. - Não foi necessário o uso de animais para treinamento das técnicas, anulando totalmente a possibilidade de estresse para esses animais. $(3,3 \%)$

7. - O crânio revestido para treinamento com otoscópio foi uma ideia genial! $(3,3 \%)$

8. - Melhora o manuseio do otoscópio. (3,3\%)

9. - A visualização dos dentes é bem clara. $(3,3 \%)$

10. - Mostra a dificuldade desse tipo de exame oral. $(3,3 \%)$

11. - Alta fidelidade, fácil de manusear e prático. $(3,3 \%)$

12. - Três alunos não responderam. (10\%)

13. Pontos negativos

14. - Não é possível simular a realidade com relação ao comportamento do animal. $(13,3 \%)$

15. - Impossível praticar a contenção do paciente. $(6,6 \%)$

16. - Poderia ter o corpo também, além da cabeça, para que pudéssemos praticar também a contenção dos animais. $(6,6 \%)$

17. - Vinte e dois alunos não responderam. $(73,3 \%)$

\section{DISCUSSÃO}

Para 0 treinamento dos estudantes, foram usados modelos de ensino como métodos alternativos. Esses métodos têm por objetivo substituir ou reduzir o número de animais utilizados (Presgrave, 2002). 
Não foi encontrado na literatura um modelo para o treinamento da avaliação da cavidade oral de roedores e lagomorfos, assim, acreditamos que o nosso modelo seja o primeiro.

A utilização de modelos no ensino da Medicina Veterinária pode diminuir ou, em alguns casos, substituir por completo o uso de animais vivos, quase sempre sem causar prejuízo ao aprendizado (Presgrave, 2002; Diniz et al, 2006; Klaphake, 2006). O aprendizado com modelos fornece aos alunos uma oportunidade de aprimoramento de suas habilidades, já que é possível repetir a ação várias vezes. Isso dará mais segurança para o aluno quando ele precisar realizar aquela mesma ação em um animal vivo (Diniz et al, 2006). Isso foi observado no questionário aplicado nesse estudo, em que $26 \quad(87 \%)$ dos estudantes responderam se sentir mais confiantes para avaliar a cavidade oral de um animal vivo após a aula prática com modelos. A confiança se dá pela repetição e também pelo realismo do modelo, pela facilidade de compreensão da técnica de diagnóstico e pela possibilidade de estudo da anatomia dentária. Todos os estudantes concordaram que o modelo é realista e que é possível de estudar a anatomia e a técnica de diagnóstico com o uso do modelo.

Quanto ao uso de outras técnicas para ensino, além dos modelos existe o uso de cadáveres e o uso complementar de animais vivos. Ainda há certa resistência por parte de estudantes e professores ao uso de modelos de ensino como substituto ao uso de animais (Diniz et al, 2006). No presente trabalho este fato foi verificado no questionário aplicado. 28 dos 30 estudantes $(93 \%)$ responderam que além da aula teórica, o melhor para o aprendizado seria a combinação das três técnicas: modelos, cadáveres e animais vivos.
Os pontos positivos citados pelos estudantes reforçam a ideia de que o treinamento em um modelo pode ser encorajador para o uso da técnica posteriormente em animais vivos. A observação do modelo de resina também foi colocado como um ponto positivo para o estudo da anatomia da cavidade oral desses animais.

Como limitações deste estudo podemos citar o pequeno número de estudantes que participaram da pesquisa, e o fato de não terem sido usados cadáveres para comparação durante a aula. De acordo com os comentários dos estudantes, seria interessante poder contar com um modelo animal com corpo além da cabeça, para que os alunos pudessem treinar a correta contenção desses animais juntamente com a técnica de observação da cavidade oral. Além disso, ter modelos para avaliação da cavidade oral com otoscópio em que existam alterações odontológicas também seria enriquecedor para os estudantes. De qualquer forma, foi possível observar nesse estudo uma empolgação por parte dos estudantes participantes, 0 que traz grande motivação para 0 trabalho com os métodos alternativos de ensino.

Pela importância do estudo da odontologia veterinária como especialidade nesse grupo específico de animais, sugerimos que o uso deste tipo de modelos tenha aplicação útil na rotina das universidades.

\section{CONCLUSÃO}

Este estudo estabeleceu o desenvolvimento de modelos alternativos para treinamento da avaliação da cavidade oral de roedores e lagomorfos e forneceu evidências acerca de seu valor no ensino. Pudemos observar uma excelente aceitação dos modelos por parte dos alunos, que os consideraram didáticos, 
realistas e que os ajudaram a compreender um novo assunto.

\section{AGRADECIMENTOS}

Ao Médico Veterinário Residente em Medicina Zoológica do HV-UFPR André Saldanha e ao Mestrando do Programa de Pós-graduação em Ciências Veterinárias Flávio Freitag, pela ajuda com as fotos do artigo.

\section{NOTAS INFORMATIVAS}

Protocolo 086_2018 da Comissão de Ética no Uso de Animais (CEUA) da Universidade Federal do Paraná.

\section{REFERÊNCIAS}

BÖHMER, E.; CROSSLEY, D. Objective interpretation of dental disease in rabbits, guinea pigs and chinchillas. European Journal of Companion Animal Practice. v. 21, n.1, p.47-56, 2011.

CAPELLO, V. Diagnostic imaging of dental disease in pet rabbits and rodents. Veterinary Clinic of Exotic Animals, v.19, n.3, p.757-782, 2016.

CAPELLO, V. Diagnosis and treatment of dental disease in pet rodents. Journal of Exotic Pet Medicine, v.17, n.2, p.114-123, 2008.

DINIZ, R; DUARTE, A.L.A.; OLIVEIRA, C.A.S. et al. Animais em aulas práticas: podemos substituí-los com a mesma qualidade de ensino?, Revista Brasileira de Educação Médica. v.30, n.2, p.31-41, 2006.

JENKINS, J.R. Diseases of geriatric guinea pigs and chinchillas. Veterinary Clinic of Exotic Animals, v.13, n.1, p.85-93, 2010.

KLAPHAKE, E. Common rodent procedures, Veterinary Clinic of Exotic Animals, v.9, n.2, p.389-413, 2006.
LANGE, R.R.; SCHMIDT, E.M.S. Rodentia - Roedores Selvagens (Capivara, Cutia, Paca e Ouriço). In: CUBAS, Z. S. Tratado de animais selvagens: medicina veterinária. $2^{\circ}$ ed. São Paulo: Roca, 2014, Cap. 54, p. 1137-1142.

LEGENDRE, L. Anatomy and disorders of the oral cavity of guinea pigs. Veterinary Clinic of Exotic Animals, v.19, n,3, p. 825-842, 2016.

LEGENDRE, L.F.J. Oral disorders of exotic rodents. Veterinary Clinic of Exotic Animals, v.6, n.3, p.601-628, 2003.

LENNOX, A.M., Diagnosis and treatment of dental disease in pet rabbits. Journal of Exotic Pet Medicine, v.17, n.2, p.107-113, 2008.

MÜLLER, J.; CLAUSS, M.; CODRON, D. et al. Tooth lenght and incisal wear and growth in guinea pigs (Cavia porcellus) fed diets of differente abrasiveness. Journal of Animal Physiology and Animal Nutrition, v.99, n.3, p.591-604, 2015.

PRESGRAVE, O. A. F. Alternativas para animais de laboratório: do animal ao computador. In: ANDRADE, A.; PINTO, S. C.; OLIVEIRA, R. S. (Orgs.). Animais de laboratório: criação e experimentação. Rio de Janeiro: Editora Fiocruz, 2002. $1^{\circ}$ ed., cap. 42, p. 362-367.

REITER, A.M. Pathophysiology of Dental Disease in the Rabbit, Guinea Pig and Chinchilla. Journal of Exotic Pet Medicine, v.17, n.2, p.70-77, 2008.

WIGGS, R.B.; LOBPRISE, H.B. Dental and oral disease in rodents and lagomorphos. In: WIGGS, R.B.; LOBPRISE, H.B. Veterinary dentistry. Principles and practice. Philadelphia: 
Lippincott - Raven, 1997, 1ํed., cap.

17, p. 518-537. 